\title{
Accuracy Improvement for the Determination of Parameters and Voltage Drops in Busbars, Considering the Networks Power Factor
}

\author{
Kotsur M.I., Yarymbash D.S., Bezverkhnya Yu.S., and Kotsur I.M. \\ Zaporizhzhia Polytechnic National University \\ Zaporizhzhia, Ukraine
}

\begin{abstract}
The power systems must satisfy the requirements both for high reliability and efficiency. The main component of the shop power supply systems is a busbar. There have been certain engineering techniques for the estimation of parameters, voltage and power losses, characterized by having a high error. Other methods have had a significant calculation efficiency, but without allowing the voltage drop to be determined as a function of the network power factor. Therefore, the aim of this work was to develop an approach that allowed an accurate estimation of the parameters and voltage drop in trolleys, depending on the network power factor. This approach was based on the decomposition of the electromagnetic processes in a trolley busbar by connecting one phase to estimate both the resistance and reactance in the absence of the external field, and two phases to estimate the resistance and reactance in the presence of the external field. The most significant results were the determination of the resistances and reactance, depending on the frequency of the current harmonics and the distance between the phases of the busbar. The dependences were proposed to estimate the resistance and reactance for the corresponding phases and current harmonic. The analytical expression of the ratio between the voltage drop in trolleys and the power factor of the network was obtained. The testing data confirmed the high accuracy of the proposed approach. The significance of the results composed a more precise determination of the parameters and voltage drops in each phase of trolleys, irregardless of their location, number, shape, as well as the phase currents' non-symmetry, wasting no time for the field simulation.
\end{abstract}

Keywords: busbar, electromagnetic field, model, resistance, reactance, voltage drop, current harmonics, power factor.

DOI: https://doi.org/10.52254/1857-0070.2021.3-51.04

UDC: 621.316.35:006.354

\section{Creșterea preciziei de determinare a parametrilor și a pierderilor de tensiune în trolei ai conductoarelor- bare, ținând cont de factorul de putere al rețelei \\ Kotsur M.I., Yarymbash D.S., Bezverkhnya Yu.S., Kotsur I.M. \\ Zaporizhzhia Polytechnic National University, Zaporizhzhia, Ukraine}

Rezumat. Sistemele de alimentare în halele de producere trebuie să îndeplinească cerințe stricte pentru fiabilitate şi eficiență ridicate. Una dintre componentele importante ale sistemelor de alimentare cu energie ale hale de producere sunt trolei ai conductoarelor-bare. Există metode inginerești pentru determinarea parametrilor, pierderilor de tensiune și putere, dar în același timp au o eroare mare; alte metode au o eficiență ridicată de calcul, dar nu permit determinarea căderii de tensiune în funcție de factorul de putere al rețelei. Prin urmare, acest obiectiv este atins prin dezvoltarea unei abordări care permite cu o precizie ridicată să determine parametrii și căderea de tensiune în conructoarele-bare, în funcție de factorul de putere al rețelei, care se bazează pe descompunerea proceselor electromagnetice în trolei ai conductorului-bară prin deconectarea uneia dintre fazele conductorului-bară pentru determinarea rezistenței active și reactanței fără influența unui câmp magnetic extern, precum și două faze ale conductorului-bară pentru a determina rezistența activă și reactivă în prezența unui câmp magnetic extern cauzat prin acțiunea unui curent extern al condutorului-bară alăturat. Cele mai semnificative rezultate sunt determinarea în baza modelării în câmp a rezistențelor active și reactive, în funcție de frecvența armonicelor curente și distanța dintre fazele trolilor de bare. Sunt propuse dependențe pentru determinarea valorilor rezistențelor active și reactive pentru fazele corespunzătoare și armonicele curentului.

Cuvinte-cheie: conductor-bară, câmp electromagnetic, model, rezistență activă și reactivă, pierderi de tensiune, armonici de curent, factor de putere.

(C) Kotsur M.I., Yarymbash D.S.,

Berverkhnya Yu.S., Kotsur I.M., 2021 


\section{Повышение точности определения параметров и потерь напряжения в троллеях шинопровода с учетом коэффициента мощности сети \\ Коцур М.И., Ярымбаш Д.С., Безверхняя Ю.С., Коцур И.М.}

Национальный университет «Запорожская политехника»»

Запорожье, Украина

Аннотация. Системы цехового электроснабжения должны удовлетворять жестким требованиям высокой надежности и эффективности. Одним из важных компонентов систем цехового электроснабжения являются троллейные шинопроводы. Существуют инженерные методы определения параметров, потерь напряжения и мощности, но при этом имеют высокую погрешность; другие методы обладают высокой эффективностью расчета, но не позволяют определить падение напряжения в зависимости от коэффициента мощности сети. Поэтому поставленная цель достигается за счет разработки подхода, позволяющего с высокой точностью определить параметры и падение напряжений в троллеях шинопровода в зависимости от коэффициента мощности сети, который основан на декомпозиции электромагнитных процессов в троллейном шинопроводе посредством включения одной из фаз шинопровода для определения активного и реактивного сопротивлений без влияния внешнего магнитного поля, а также двух фаз шинопровода для определения активного и реактивного сопротивлений при наличии внешнего магнитного поля, вызванного действием стороннего тока соседнего троллея шинопровода. Наиболее существенными результатами являются определение на основе полевого моделирования активных и реактивных сопротивлений, в зависимости от частоты гармоник тока и расстояния между фазами троллей шинопровода. Предложены зависимости для определения значений активного и реактивного сопротивлений для соответствующих фаз и гармоник тока. Получено аналитическое выражение зависимости падения напряжения в троллеях от коэффициента мощности сети. Данные экспериментального исследования подтверждают высокую эффективность и точность предложенного подхода. Значимость полученных результатов состоит в более точном определении параметров и потерь напряжения в каждой фазе троллеев шинопровода не зависимо от их расположения, количества, формы, а также не симметрии фазных токов без затрат времени на полевое моделирование.

Ключевые слова: шинопровод, электромагнитное поле, модель, активное и реактивное сопротивление потери напряжения, гармоники тока; коэффициент мощности.

\section{I. ВВЕДЕНИЕ}

Системы цехового электроснабжения должны удовлетворять современным требованиям высокой надежности, энергоэффективности и энергобезопасности, обеспечивая при этом высокое качество электрической энергии, не зависимо от режимов работы электроприемников. Современные системы цехового электроснабжения могут иметь значительную протяженность порядка 700-1000 м и более. В качестве основных токоведущих элементов для систем цехового электроснабжения являются крановые и станочные троллейные шинопроводы. Поэтому, их параметры (активное и индуктивное сопротивления) и электромагнитные характеристики оказывают существенное влияние на качество электроэнергии, режимы работы электроприемников, энергоэффективность технологических процессов, следовательно, на себестоимость конечной продукции предприятий [1]. Наличие силового электрооборудования, к которым относятся современные системы электропривода мостовых кранов, станков с ЧПУ [2], выпрямителей и других полупроводниковых преобразователей [3], трансфор- маторов с насыщенными магнитными системами [4] и др. оказывает существенное влияние на гармонический состав токов и напряжений в цеховой электросети [5]-[6]. Следствием наличия высших гармоник тока является существенное увеличение активных и реактивных сопротивлений троллей шинопровода, потерь напряжения и активных потерь, которые приводят к снижению напряжения в точке подключения электроприемников ниже своего номинального значения, а также к снижению коэффициента мощности сети [7]. В [8]-[9] предложены методики выбора конфигурации сетей и расчета параметров стальных и медных троллей шинопровода. Они основаны на обобщенных уравнениях и имеют вид эмпирических зависимостей, которые не позволяют учесть влияние высших гармоник тока на параметры фазных троллеев шинопровода, падение напряжения, активных потерь, не симметрию электромагнитных параметров, а также скин-эффект, эффекты близости, а также краевые эффекты, что в общем счете может привести к большим погрешностям. В [10] выполнено исследование влияния высших гармоник тока на потери напряжения и мощности в токопроводах си- 
стем питания кранов. Получены необходимые расчетные соотношения для определения параметров токопроводов при наличии высших гармоник тока. Однако эти результаты было получены с допущением отсутствия влияния скин-эффекта, эффекта близости, поверхностных и других краевых эффектов. В [11] представлен метод дискретных численных расчетов для определения распределений магнитного поля в сильноточных прямоугольных шинах большой протяженности. Этот метод основан на методе эквивалентной схемы частичного элемента, и реализуется с помощью интегральных уравнений. Он учитывает скин-эффект и эффекты близости, а также полную электромагнитную связь между фазными шинами и нейтральной шиной. В частности, описаны магнитные поля в прямоугольных фазных и нейтральных сборных шинах неэкранированных трехфазных систем. В [12] предложен новый численный метод расчета импедансов системы прямоугольных сборных шин. Этот метод основан на теории частичной индуктивности. В частности, описан импеданс трехфазной системы прямоугольных сборных шин с нейтральной сборной шиной. Были получены результаты для активного и реактивного сопротивления для этой системы с несколькими прямоугольными проводниками, а также были приняты во внимание скин-эффекты и эффекты близости. Данные методы [11], [12] применимы только для прямоугольных шин при частоте тока 60Гц. В [13] разработан эффективный численный расчет импедансов прямоугольных линий передачи. Метод реализуется с помощью интегральных уравнений для плотности тока, где поперечное сечение прямоугольного токопровода дискретизируется для улучшения сходимости с помощью неоднородной расчетной области. Проведено исследование распределения плотности тока и его влияния на импеданс, особенно для высокочастотного диапазона.

Для учета влияния геометрической формы, особенностей расположения и расстояний между троллеями шинопровода, нелинейности свойств их активных материалов, скин-эффектов, эффектов близости, поверхностных и других краевых эффектов, могут быть применены методы расчета электромагнитных параметров троллейного шинопровода на основе полевого моделирования [14][15]. Данные методы получили широкое распространение для широкого класса задач электротехники [16]-[18].

Однако их реализация на основе методов конечных элементов с учетом нелинейных электрофизических свойств, гармонического состава токов осложнена большими затратами времени на численную реализацию из-за необходимости сходимости расчета для каждого временного слоя [19]. В работе [20] была предложена двухмерная полевая модель в частотной формулировке распределения электромагнитного поля, которая позволяет значительно снизить время расчета на численную реализацию и позволит учесть в отдельности влияние каждой гармонической составляющей тока на параметры и электромагнитные характеристики электротехнических систем. Для учета нелинейности магнитных свойств данных моделей применяются эффективные значения магнитных свойств ферромагнитных материалов [21]. В ранних работах авторов статьи, на основании результатов полевого моделирования получена функциональная зависимость в виде бикубического полинома, которая позволяет для действующих спектров и амплитуд высших гармоник тока, а также коэффициента короткого замыкания, при выборе соответствующих коэффициентов полинома, определить составляющие и результирующие значения падений напряжения для конкретного шинопровода без затрат времени на полевое моделирование. Недостатком двухмерной полевой модели в частотной формулировке распределения электромагнитного поля и данного подхода по определению составляющих и результирующих значений падений напряжения без затрат времени на полевое моделирование является то, что рассчитанные значения параметров троллей шинопровода и падения напряжения в них справедливы только для коэффициента мощности сети равного единице.

Таким образом, целью работы является разработка подхода, основанного на использовании полевых моделей, позволяющего с высокой точностью и эффективностью для основной и высших гармоник тока, с учетом, скин-эффекта, эффекта близости, краевых, поверхностных и других эффектов, нелинейности магнитных и электрофизических свойств материалов, не симметрии фазных токов в троллеях шинопровода, а также их количества, определить их параметры, а также падения напряжений в зависимости от ко- 
эффициента мощности сети.

\section{II. МЕТОД ИССЛЕДОВАНИЯ}

Основная идея нового похода заключается в определении индуктивностей рассеивания и активного сопротивления с учетом влияния скин-эффекта и поверхностного эффекта, при отсутствии внешнего магнитного поля, а также взаимных индуктивностей и активного сопротивления при наличии внешнего поля, вызванного сторонними токами в соседних троллеях шинопровода (эффект близости). При этом принимается допущение о справедливости использования метода суперпозиции для определения индуктивностей стальных троллеев шинопровода, имеющие нелинейные магнитные свойства.

Известно, что подмагничивающим свойством обладает фазный ток соседнего токопровода с опережающей фазой, размагничивающим - ток с отстающей фазой. Влияние этих процессов приводит к несимметрии системы взаимных индуктивностей, а также активных сопротивлений между фазами токопровода. Поэтому для определения индуктивностей рассеивания и взаимных индуктивностей, а также активного сопротивления, с учетом и без учета влияния эффекта близости для каждой отдельной $k$-ой гармоники фазного тока, рассматриваются случаи раздельного включения каждой фазы А, B, C шинопровода, а также группового включения двух фаз троллеев шинопровода, которое выполняется с помощью полевого моделирования.

Фазные троллеи шинопровода имеют идентичное геометрическое строение и размеры, следовательно, активное сопротивление и индуктивности рассеяния, поэтому для их определения достаточно включения одной из троллеев шинопровода.

Для определения взаимной индуктивности и активного сопротивления в троллеи шинопровода, с учетом влияния эффекта близости, выполняется групповое включение двух фазных троллей.

Причем, для первой троллеи принимается условие, что вектор тока условной фазы А имеет фиксированное значение, которое соответствует собственным значениям амплитуд для $k$-х гармоник тока.

Для второй троллеи, которая геометрически расположена на месте троллеи условной фазы В, угол вектора фазного тока $\varphi_{B}$ изменяется на полный период при соответствующей $k$-ой гармонике тока. Для третьей троллеи, которая геометрически расположена на месте троллеи условной фазы C, угол вектора фазного тока $\varphi_{C}$ также изменяется на полный период относительно вектора тока фазы А первой троллеи шинопровода, для соответствующей $k$-ой гармоники тока. Для многофазного шинопровода четвертая, пятая и т.д. троллеи смещаются на тоже расстояние, что и троллеи условной фазы $\mathrm{B}$, относительно условной фазы А. Это позволит получить зависимости полной индуктивности и активного сопротивления фазной троллеи при учете влияния эффекта близости соседних фазных троллеев шинопровода, в зависимости от их расстояния и количества.

При изменении вектора тока на полный его период, протекающего в соседнем троллее условной фазы $\mathrm{B}$, активное сопротивление троллеи условной фазы А будет изменяться по периодическому закону:

$$
\begin{gathered}
\left.R_{k}\right|_{A}=\left.R_{k}\right|_{A A}-\left.R_{k}\right|_{A B}=\left.R_{k}\right|_{A A}- \\
-\left.R_{m k}\right|_{A B} \sin \cdot\left(\frac{\pi \cdot \varphi_{1}}{180}+\frac{\alpha \cdot \pi}{180}\right), \\
\varphi_{1}=\varphi_{B}-\varphi_{A},
\end{gathered}
$$

где $\left.R_{k}\right|_{\text {AА }}$ - активное сопротивление троллеи условной фазы А (без учета влияния эффекта близости), Ом; $\left.R_{k}\right|_{A B}$ - величина изменения активного сопротивления условной фазы А при протекании тока в соседнем троллее условной фазы В (с учетом влияния эффекта близости), Ом.

При изменении вектора тока на полный его период, протекающего в соседнем троллее условной фазы $\mathrm{B}$, полная индуктивность троллеи условной фазы А будет изменяться по периодическому закону, функция которого соответствует функции вида:

$$
\begin{aligned}
& \left.\quad L_{k}\right|_{A}=\left.L_{k}\right|_{A A}+\left.M_{k}\right|_{A B}=\left.L_{k}\right|_{A A}+ \\
& +\left.M_{m k}\right|_{A B} \cdot \cos \left(\frac{\pi \cdot \varphi_{1}}{180}+\frac{\alpha \cdot \pi}{180}\right)
\end{aligned}
$$


где $\left.L_{k}\right|_{A A}$ - индуктивность рассеивания условной фазы А (собственная индуктивность), Гн/м; $\left.M_{k}\right|_{A B}$ - амплитуда взаимной индуктивности между условными фазами А и В, Гн/м.

По данным полевого моделирования строятся зависимости активного сопротивления $\left.R_{k}\right|_{A A}$ и индуктивностей рассеивания $\left.L_{k}\right|_{A A}$ от частоты $k-\mathrm{x}$ гармоник тока без учета влияния эффекта близости, а также зависимости величин изменения активного сопротивления $\left.R_{k}\right|_{A B}$ и амплитуд взаимной индукции $\left.M_{k}\right|_{A B}$ при влиянии тока в соседнем троллеи условной фазы В, в зависимости от расстояния между фазными троллеями шинопровода для соответствующих значений частоты $k$-х гармоник тока. По полученным зависимостям определяются $\left.R_{k}\right|_{A A},\left.R_{k}\right|_{A B},\left.L_{k}\right|_{A A}$ и $\left.M_{k}\right|_{A B}$ для каждой соответствующей фазы, в зависимости от их расположения между собой, а также угла между векторами фазных токов для соответствующих $k$-х гармоники тока.

Таким образом, для трех фазной системы троллейного шинопровода, при соответствующей $k$-ой гармоники тока, можно определить значение активного сопротивления троллеи для фазы А при протекании токов в соседних троллеях фаз В и $\mathrm{C}$ :

$$
\begin{gathered}
\left.R_{k}\right|_{A}=\left.R_{k}\right|_{A A}-\left.R_{k}\right|_{A B}-\left.R_{k}\right|_{A C}=\left.R_{k}\right|_{A A}- \\
-\left.R_{m k}\right|_{A B} \cdot \sin \left(\frac{\pi\left(\varphi_{B}-\varphi_{A}\right)}{180}+\frac{\alpha \cdot \pi}{180}\right)- \\
-\left.R_{m k}\right|_{A C} \cdot \sin \left(\frac{\pi\left(\varphi_{C}-\varphi_{A}\right)}{180}+\frac{\alpha \cdot \pi}{180}\right)
\end{gathered}
$$

для фазы B -

$$
\begin{gathered}
\left.R_{k}\right|_{B}=\left.R_{k}\right|_{B B}-\left.R_{k}\right|_{B C}-\left.R_{k}\right|_{B A}=\left.R_{k}\right|_{B B}- \\
-\left.R_{m k}\right|_{B C} \cdot \sin \left(\frac{\pi\left(\varphi_{C}-\varphi_{B}\right)}{180}+\frac{\alpha \cdot \pi}{180}\right)-
\end{gathered}
$$

$$
-\left.R_{m k}\right|_{B A} \cdot \sin \left(\frac{\pi\left(\varphi_{A}-\varphi_{B}\right)}{180}+\frac{\alpha \cdot \pi}{180}\right)
$$

для фазы C -

$$
\begin{gathered}
\left.R_{k}\right|_{C}=\left.R_{k}\right|_{C C}-\left.R_{k}\right|_{C A}-\left.R_{k}\right|_{C B}=\left.R_{k}\right|_{C C}- \\
-\left.R_{m k}\right|_{C A} \cdot \sin \left(\frac{\pi\left(\varphi_{A}-\varphi_{C}\right)}{180}+\frac{\alpha \cdot \pi}{180}\right)- \\
-\left.R_{m k}\right|_{C B} \cdot \sin \left(\frac{\pi\left(\phi_{B}-\phi_{C}\right)}{180}+\frac{\alpha \cdot \pi}{180}\right)
\end{gathered}
$$

Активные сопротивления для всех фазных троллеев без учета эффекта близости будут одинаковые $\left.R_{k}\right|_{A A}=\left.R_{k}\right|_{B B}=\left.R_{k}\right|_{C C}$, а также величины изменения активного сопротивления при влиянии эффекта близости между соответствующими фазами троллей: $\left.\quad R_{k}\right|_{A B}=\left.R_{k}\right|_{B A} ;\left.\quad R_{k}\right|_{A C}=\left.R_{k}\right|_{C A}$; $\left.R_{k}\right|_{B C}=\left.R_{k}\right|_{C B}$. Величины $\left.R_{k}\right|_{A B},\left.R_{k}\right|_{A C},\left.R_{k}\right|_{B C}$ формируют не симметрию активных сопротивлений $\left.R_{k}\right|_{A},\left.\left.\quad R_{k}\right|_{B} \cdot R_{k}\right|_{C}$ в фазных троллеях шинопровода.

Также для трех фазной системы троллейного шинопровода для каждой $k$-ой частоты гармоники тока можно определить значение полной индуктивности троллеи фазы А при протекании токов в соседних троллеях фаз В и С:

$$
\begin{gathered}
\left.L_{k}\right|_{A}=\left.L_{k}\right|_{A A}+\left.M_{k}\right|_{A B}+\left.M_{k}\right|_{A C}=\left.L_{k}\right|_{A A}+ \\
+\left.M_{m k}\right|_{A B} \cdot \cos \left(\frac{\pi\left(\varphi_{B}-\varphi_{A}\right)}{180}+\frac{\alpha \cdot \pi}{180}\right)+ \\
+\left.M_{m k}\right|_{A C} \cdot \cos \left(\frac{\pi\left(\varphi_{C}-\varphi_{A}\right)}{180}+\frac{\alpha \cdot \pi}{180}\right)
\end{gathered}
$$

для фазы B -

$$
\begin{gathered}
\left.L_{k}\right|_{B}=\left.L_{k}\right|_{B B}+\left.M_{k}\right|_{B C}+\left.M_{k}\right|_{B A}=\left.L_{k}\right|_{B B}+ \\
+\left.M_{m k}\right|_{B C} \cdot \cos \left(\frac{\pi\left(\varphi_{C}-\varphi_{B}\right)}{180}+\frac{\alpha \cdot \pi}{180}\right)+ \\
+\left.M_{m k}\right|_{B A} \cdot \cos \left(\frac{\pi\left(\varphi_{A}-\varphi_{B}\right)}{180}+\frac{\alpha \cdot \pi}{180}\right)
\end{gathered}
$$

для фазы C -

$$
\left.L_{k}\right|_{C}=\left.L_{k}\right|_{C C}+\left.M_{k}\right|_{C A}+\left.M_{k}\right|_{C B}=\left.L_{k}\right|_{C C}+
$$




$$
\begin{aligned}
& +\left.M_{m k}\right|_{C A} \cdot \cos \left(\frac{\pi\left(\varphi_{A}-\varphi_{C}\right)}{180}+\frac{\alpha \cdot \pi}{180}\right)+ \\
& +\left.M_{m k}\right|_{C B} \cdot \cos \left(\frac{\pi\left(\varphi_{B}-\varphi_{C}\right)}{180}+\frac{\alpha \cdot \pi}{180}\right)
\end{aligned}
$$

Индуктивности рассеивания для всех фазных троллеев будут одинаковые $\left.L_{k}\right|_{A A}=\left.L_{k}\right|_{B B}=\left.L_{k}\right|_{C C}, \quad$ а также взаимные индуктивности, формирующие связь между фазами троллеев шинопровода: $\left.M_{k}\right|_{A B}=\left.M_{k}\right|_{B A} ;$ $\left.M_{k}\right|_{A C}=\left.M_{k}\right|_{C A} ;$ $\left.M_{k}\right|_{B C}=\left.M_{k}\right|_{C B}$. Взаимные индуктивности $\left.M_{k}\right|_{A B},\left.\quad M_{k}\right|_{B C},\left.\quad M_{k}\right|_{A C}$ формируют не симметрию полных индуктивностей $\left.L_{k}\right|_{A}$, $\left.L_{k}\right|_{B},\left.L_{k}\right|_{C}$ в фазных троллеях, шинопровода.

Значения полного реактивного сопротивления для каждой $k$-ой гармоники тока, для соответствующей фазы троллеев шинопровода, можно определить из следующей системы уравнений [2]:

$$
\left.\begin{array}{l}
\left.X_{k}\right|_{A}=\left.2 \cdot \pi \cdot f_{k} \cdot L_{k}\right|_{A} \\
\left.X_{k}\right|_{B}=\left.2 \cdot \pi \cdot f_{k} \cdot L_{k}\right|_{B} \\
\left.X_{k}\right|_{C}=\left.2 \cdot \pi \cdot f_{k} \cdot L_{k}\right|_{C}
\end{array}\right\} .
$$

Также, как и в случае с активным и реактивным сопротивлениями, при изменении вектора тока на полный его период, протекающего в соседнем троллее условной фазы $\mathrm{B}$, потери напряжения в условной фазе А будут также меняться по периодическому закону, функция которого соответствует функции вида:

$$
\begin{aligned}
\left.\Delta U_{k}\right|_{A B} & =\left.\Delta U_{k}\right|_{A A}+\left.\Delta U_{k}\right|_{A B}=\left.\Delta U_{k}\right|_{A A}+ \\
& +\left.\Delta U_{m k}\right|_{A B} \sin \cdot\left(\frac{\pi \cdot \varphi_{1}}{180}+\frac{\alpha \cdot \pi}{180}\right),
\end{aligned}
$$

где $\left.\Delta U_{k}\right|_{A A}$ - потери напряжения от тока фазы $\mathrm{A}, \mathrm{B} / \mathrm{M} ;\left.\quad \Delta U_{k}\right|_{A B}$ - составляющая потерь напряжения от наличия наведенной ЭДС, вызванной действием стороннего тока в троллеи условной фазы В, В/м.

Периодические функции, обеспечивающие зависимость изменения падения напряжения
(11) и активного сопротивления (1) от угла между векторами токов условных фаз А и В троллей шинопровода, будут имеет между собой сдвиг по фазе на угол $\varphi_{T}$, значение которого соответствует отношению реактивного сопротивления к активному сопротивлению для соответствующих фаз и частоты $k$-ой гармоники тока:

$$
\left.\phi_{T k}\right|_{A, B, C}=\left.\operatorname{arctg}\left(\frac{X_{k}}{R_{k}}\right)\right|_{A, B, C} .
$$

По аналогии (4) - (6) для активного сопротивления и (7) - (9) для полной индуктивности для каждой $k$-ой частоты гармоники тока напишем уравнение падения напряжения в троллее фазы А при действии внешнего электромагнитного поля, созданного токами в троллеях условных фаз В и С:

$$
\begin{gathered}
\left.\Delta U_{k}\right|_{A}=\left.\Delta U_{k}\right|_{A A}+\left.\Delta U_{k}\right|_{A B}+\left.\Delta U_{k}\right|_{A C}=\left.\Delta U_{k}\right|_{A A}+ \\
+\left.\Delta U_{m k}\right|_{A B} \cdot \sin \left(\frac{\pi\left(\varphi_{B}-\varphi_{A}+\left.\varphi_{T k}\right|_{A}\right)}{180}+\frac{\alpha \cdot \pi}{180}\right)+ \\
+\left.\Delta U_{m k}\right|_{A C} \cdot \sin \left(\frac{\pi\left(\varphi_{C}-\varphi_{A}+\left.\varphi_{T k}\right|_{A}\right)}{180}+\frac{\alpha \cdot \pi}{180}\right)
\end{gathered}
$$

для фазы B -

$$
\begin{gathered}
\left.\Delta U_{k}\right|_{B}=\left.\Delta U_{k}\right|_{B B}+\left.\Delta U_{k}\right|_{B C}+\left.\Delta U_{k}\right|_{B A}=\left.\Delta U_{k}\right|_{B B}+ \\
+\left.\Delta U_{m k}\right|_{B C} \cdot \sin \left(\frac{\pi\left(\varphi_{C}-\varphi_{B}+\left.\varphi_{T k}\right|_{B}\right)}{180}+\frac{\alpha \cdot \pi}{180}\right)+ \\
+\left.\Delta U_{m k}\right|_{B A} \cdot \sin \left(\frac{\pi\left(\varphi_{A}-\varphi_{B}+\left.\varphi_{T k}\right|_{B}\right)}{180}+\frac{\alpha \cdot \pi}{180}\right)(14)
\end{gathered}
$$

для фазы $\mathrm{C}$ -

$$
\begin{gathered}
\left.\Delta U_{k}\right|_{C}=\left.\Delta U_{k}\right|_{C C}+\left.\Delta U_{k}\right|_{C A}+\left.\Delta U_{k}\right|_{C B}=\left.\Delta U_{k}\right|_{C C}+ \\
+\left.\Delta U_{m k}\right|_{C A} \cdot \sin \left(\frac{\pi\left(\varphi_{A}-\varphi_{C}+\left.\varphi_{T k}\right|_{C}\right)}{180}+\frac{\alpha \cdot \pi}{180}\right)+ \\
+\left.\Delta U_{m k}\right|_{C B} \cdot \sin \left(\frac{\pi\left(\varphi_{B}-\varphi_{C}+\left.\varphi_{T k}\right|_{C}\right)}{180}+\frac{\alpha \cdot \pi}{180}\right)(15
\end{gathered}
$$

Если на параметры шинопровода не влияют значения амплитуд $k$-х гармоник тока, и определение их параметров для 
каждой $k$-ой частоты гармоники тока не является сложной задачей, то для определения потерь напряжения в троллеях фаз А, B, С, для которых значения амплитуд $k$-х гармоник тока прямолинейно влияют на их результирующее значение и является очень сложной и трудоемкой задачей, при решении которой необходимо заранее учитывать режимы работы цеховых токоприемников. При этом, данный подход позволяет определять потери напряжения только при коэффициенте мощности сети $\cos (\varphi)=1$. Поэтому для упрощения задачи, относительно определения потерь напряжения и учета $\cos (\varphi)$, в зависимости от характера работы цеховых токоприемников, предлагается усовершенствовать известное уравнение [8]-[9]:

$$
\begin{gathered}
\left.\Delta U_{k}\right|_{A, B, C}=\left\lfloor\left.\left(R_{k} \cdot \cos \varphi\right)\right|_{A, B, C}+\right. \\
\left.+\left.\left(X_{k} \cdot \sin \varphi\right)\right|_{A, B, C}\right\rfloor\left.\cdot I_{H, k}\right|_{A, B, C} \cdot l .
\end{gathered}
$$

С учетом сдвига по фазе на угол $\phi_{T}$, падение напряжения относительно активного сопротивления, для соответствующей $k$-ой гармоники тока и фазы шинопровода, можно представить в виде:

$$
\begin{gathered}
\left.\Delta U_{k}\right|_{A, B, C}=\left\lfloor\left.\left(R_{k} \cdot \cos \left(\varphi+\phi_{T k}\right)\right)\right|_{A, B, C}+\right. \\
\left.+\left.\left(X_{k} \cdot \sin \left(\varphi+\phi_{T k}\right)\right)\right|_{A, B, C}\right\rfloor\left.\cdot \quad I_{H, k}\right|_{A, B, C} \cdot l
\end{gathered}
$$

где $\left.\Delta U_{k}\right|_{A, B, C}$ - потери напряжения для соответствующей $k$-ой гармоники тока, для фаз А,B,C; $\left.\quad I_{H, k}\right|_{A, B, C} \quad$ - ток нагрузки соответствующей $k$-ой гармоники, для фаз $\mathrm{A}, \mathrm{B}, \mathrm{C} ; l$ - длина троллеи от точки питания до точки приложения нагрузки, м.

Или в следующем виде:

$$
\begin{gathered}
\left.\Delta U_{k}\right|_{A, B, C}=\left[\left(R_{k} \cdot \cos \phi_{T k}+X_{k} \cdot \sin \phi_{T k}\right) \times\right. \\
\times \cos \varphi-\left(R_{k} \cdot \sin \phi_{T k}-\right. \\
\left.\left.-X_{k} \cdot \cos \phi_{T k}\right) \cdot \sin \varphi\right]\left._{A, B, C} \cdot I_{H, k}\right|_{A, B, C} \cdot l
\end{gathered}
$$

Так как шинопроводы имеют различную геометрию троллей, их количество, расстояние между ними, а также разновидность материалов, из которых они изготовлены, для каждого вида шинопровода разрабатывается полевая модель в частотной постановке распределения электромагнитного поля, учитывающая конструктивные особенности, нелинейность электрофизических и магнитных свойств активных и конструкционных материалов шинопровода, скин-эффекты, эффекты близости, а также поверхностные и другие краевые эффекты. Система уравнений Максвелла формулируется для комплексных амплитуд векторного магнитного и электрического потенциалов в геометрических электропроводящих областях и окружающей их электроизоляционной среды, согласно [15]:

$$
\left\{\begin{array}{c}
-\nabla \cdot\left[\left(j \omega \sigma_{i}-\omega^{2} \varepsilon_{0} \varepsilon_{r, i}\right) \cdot \boldsymbol{A}_{i-k}+\right. \\
\left.+\left(\sigma_{i}+j \omega \varepsilon_{0} \varepsilon_{r, i}\right) \nabla V_{i, k}-\boldsymbol{J}_{i, k}^{\boldsymbol{e}}\right]=0 ; \\
\left(j \omega \sigma_{i}-\omega^{2} \varepsilon_{0} \varepsilon_{r, i}\right) \boldsymbol{A}_{i, k}+\nabla \times\left(\mu_{0}^{-1} \mu_{r, i}^{-l} \nabla \times \boldsymbol{A}_{i, k}\right)+ \\
+\left(\sigma_{i}+j \omega \varepsilon_{0} \varepsilon_{r, i}\right) \nabla V_{i, k}=\boldsymbol{J}_{i, k}^{\boldsymbol{e}} ;
\end{array}\right.
$$

где $\omega-$ угловая частота, рад/с; $\sigma$ - электрическая проводимость, См/м; $\varepsilon_{0}-$ электрическая постоянная, Ф/м; $\varepsilon_{r}$ - диэлектрическая проницаемость; $\boldsymbol{A}$ - векторный магнитный потенциал, Вб/м; $V$ - комплексная амплитуда электрического потенциала, В; $\boldsymbol{J}^{\boldsymbol{e}}-$ комплексная плотность тока, $\mathrm{A} / \mathrm{M}^{2} ; \mu_{0}-$ магнитная постоянная, Гн/м; $\mu_{r}$ - эффективная магнитная проницаемость; индекс $i$ - элемент конструкции шинопровода; индекс $k$ - соответствует порядковому номеру гармоники тока.

По данным численного моделирования определяется энергия магнитного поля, активные потери в расчетной $i$-й области при соответствующих значениях амплитуды и частоты $k$-й гармоники тока, активные, реактивные и полные электрические сопротивления троллей каждой из фаз, а также падение напряжения в троллеях шинопровода [15].

По данным полевого моделирования строятся функциональные зависимости для каждой $k$-ой гармоники тока для определения активного и реактивного сопротивлений, с учетом и без учета влияния эффекта близости, в зависимости от расстояния между троллеями шинопровода.

Полученные зависимости в дальнейшем для конкретного шинопровода, без затрат времени на полевое моделирование, могут быть использованы в инженерной практике, что позволит эффективно подобрать конфигурацию сети цехового электроснабжения, определить, места установки дополнительных подпиточных секций и шин, точно определить 
установленную мощность фильтрующих и компенсирующих устройств, что даст возможность повысить качество и надежность работы электроприемников с заявленными паспортными данными.

\section{III. АНАЛИЗ И РЕЗУЛЬТАТЫ ИССЛЕДОВАНИЯ}

Моделирование выполнялось для стальных троллей шинопровода уголковой формы для профиля размером $30 \times 30 \times 4$, расстояние между фазными троллеями $l=13$ мм, при значении номинального тока основной гармоники $I_{\phi}=50 \mathrm{~A}$ и амплитудами и частотами для каждой $k$-ой гармоники тока согласно [22], при коэффициенте короткого замыкания $R_{s c e}=33$ о.е. На рис. 1 приведены результаты полевого моделирования электромагнитных процессов в троллеях шинопровода для частоты $f_{k}=50$ и 650 Гц при соответствующих значениях амплитуд гармоник тока.

На основе данных полевого моделирования получены зависимости активного сопротивления и индуктивности рассеяния без учета влияния эффекта близости между соседними троллеями шинопровода от частоты $k$-ой гармоники тока (рис. 2.) на 1м длины стального троллея шинопровода. С увеличением частоты $k$-ой гармоники тока имеет место увеличение до 6,11 раз активного сопротивления $\left.R_{k}\right|_{A A}$

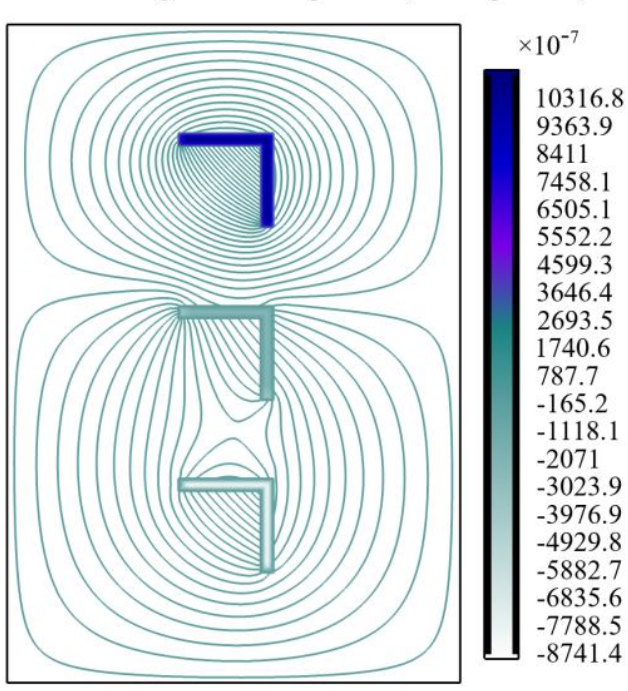

a)

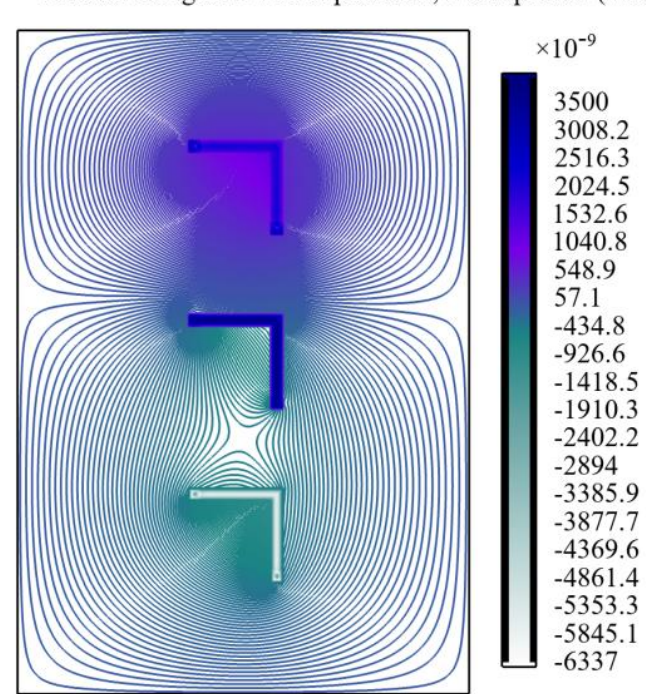

б)

Рис. 1 - Результаты двухмерного полевого моделирования для стальных троллей шинопровода уголковой формы при частоте $f_{k}=50 Г ц$ (a) и $f_{k}=650 Г ц$ (б) при соответствующих значениях амплитуды гармоник тока. ${ }^{1}$

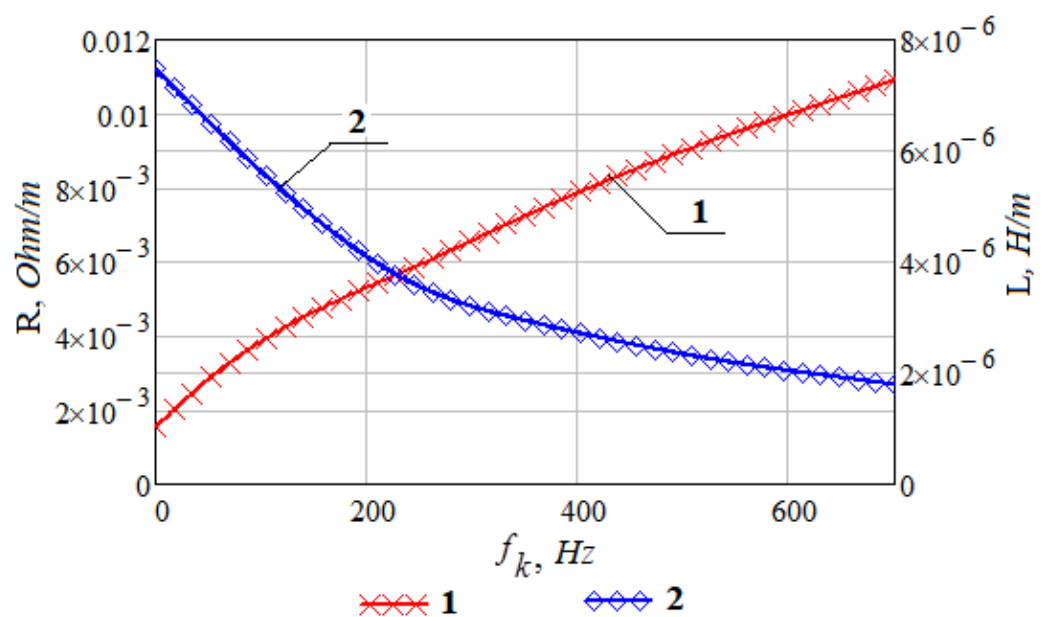

Рис. 2 - Зависимости активного сопротивления (1) и индуктивности рассеяния (2) от частоты $k$-х гармоник тока при отсутствии внешнего магнитного поля. ${ }^{2}$

1,2 Appendix 1 
троллея шинопровода относительно значения активного сопротивления при постоянном токе (при $f_{k}=0$ ). Зависимости активного сопротивления $\left.R_{k}\right|_{\text {AA }}$ стального троллея от частоты $k$-й гармоники тока имеют нелинейный характер, что вызвано влиянием скин-эффекта и поверхностного эффекта. При увеличении $f_{k}$ значение индуктивности рассеяния $\left.L_{k}\right|_{A A}$ уменьшается, так как при увеличении частоты тока уменьшается область локализации магнитного поля (рис.1).

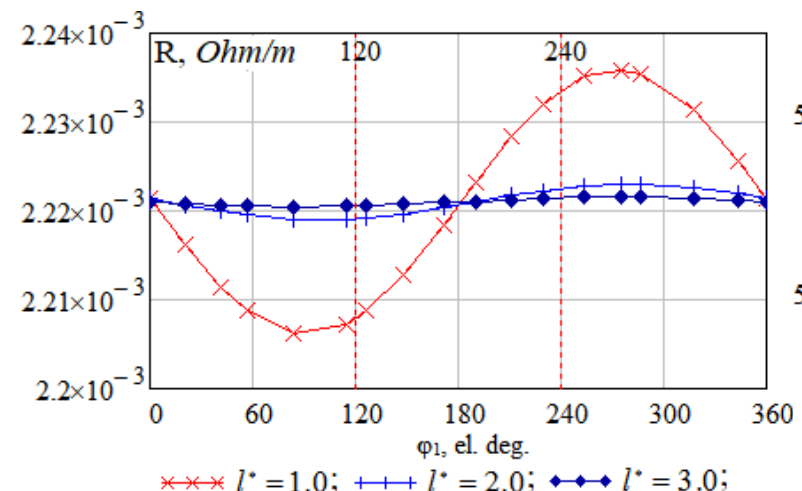

a)

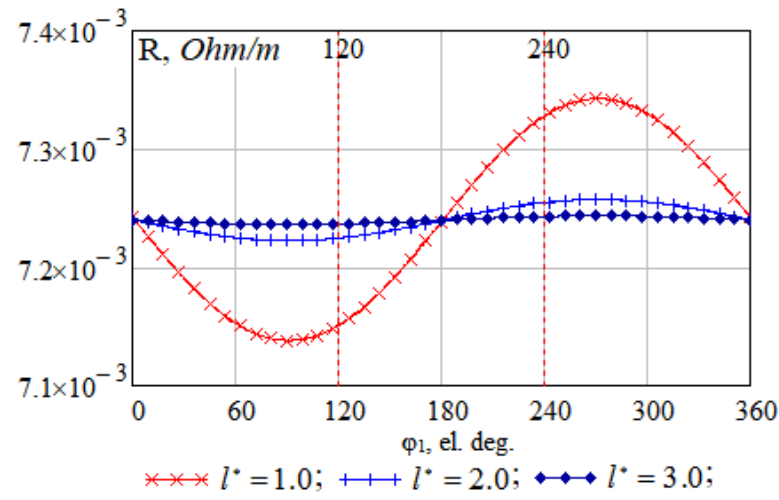

B)
На основе данных полевого моделирования получена зависимость активного сопротивления $\left.R_{k}\right|_{A B}$ стального троллея условной фазы А шинопровода от угла сдвига $\varphi_{1}$ вектора тока, протекающего в соседнем троллеи фазы В шинопровода для каждой $k$-ой гармоники тока при разных значениях межосевого расстояния $l^{*}$ между соседними троллеями условных фаз А и В. (рис. 3).

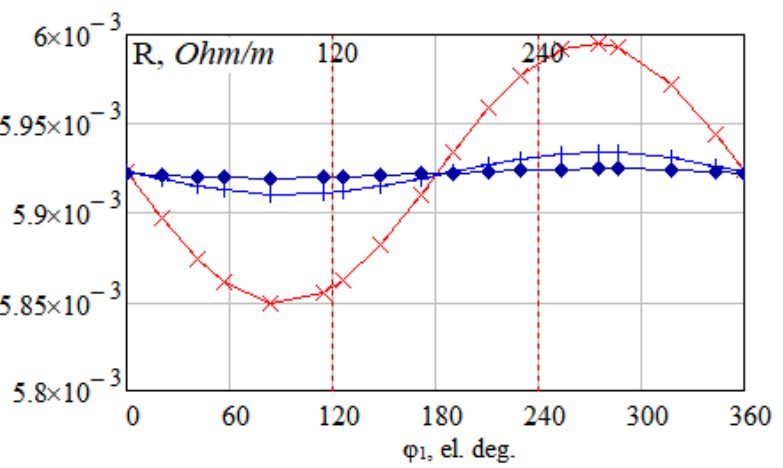

$* * * l^{*}=1.0 ;+1+l^{*}=2.0 ; \leftrightarrow l^{*}=3.0$;

б)

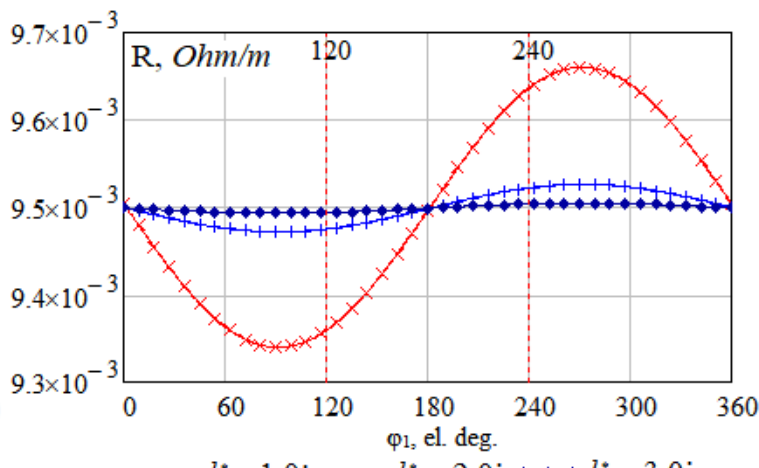

$* * * l^{*}=1.0 ;+1+l^{*}=2.0 ; \leftrightarrow l^{*}=3.0 ;$

г)

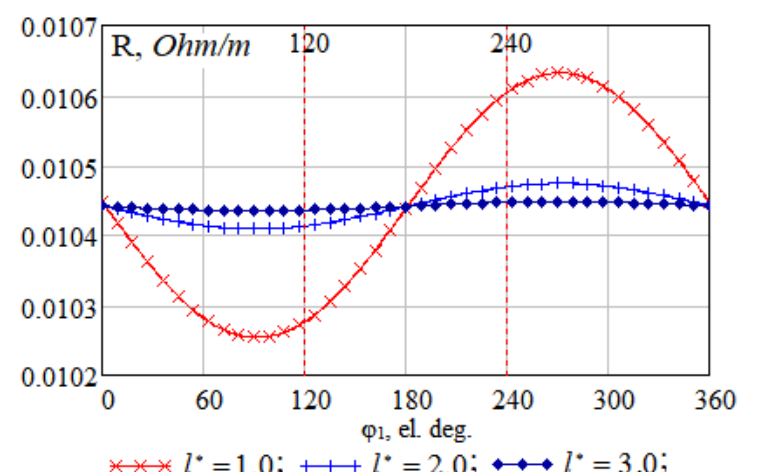

д)

Рис. 3 - Зависимости активного сопротивления стального троллея условной фазы А шинопровода от угла сдвига вектора тока, протекающего в соседнем троллее условной фазы В, для: 1-й (а), 5-й (б), 7-й (в), 11-й (г), 13-й (д) гармоник тока. ${ }^{3}$

\footnotetext{
${ }^{3}$ Appendix 1
} 
Постоянная составляющая активного сопротивления $\left.R_{k}\right|_{A}$ при наличии внешнего магнитного поля соответствует своему значению активного сопротивления троллеи условной фазы А $\left.R_{k}^{6}\right|_{A A}$ при соответствующей частоте $k$-ой гармоники тока при отсутствии внешнего магнитного поля (рис. 2). Когда вектор тока, условной фазы B, протекающего в соседних троллеях, совпадает с вектором тока условной фазы A ( $\varphi_{1}=0$ эл. град), а также когда токи условных фаз А и В протекают встречно ( $\varphi_{1}=180$ ел. град), то активные сопротивления при условии наличия $\left.R_{k}\right|_{A}$ и отсутствии внешнего магнитного поля $\left.R_{k}\right|_{A A}$ троллеи условной фазы А будут равны (см. рис. 3).

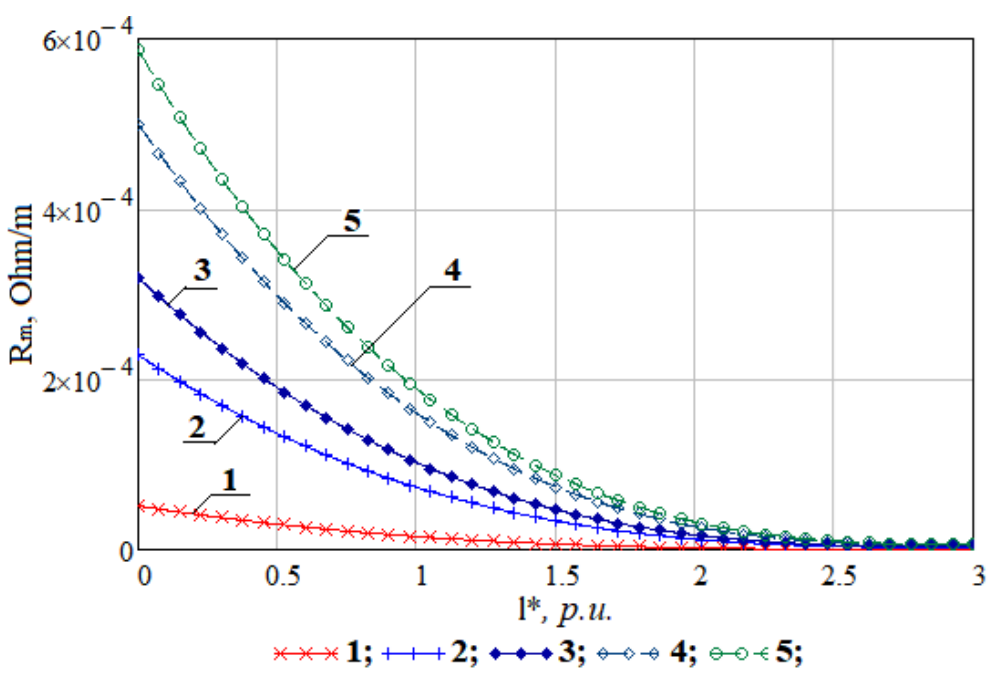

Рис. 4 - Зависимости амплитуды изменения активного сопротивления от влияния внешнего магнитного поля в зависимости от расстояния между соседними троллеями для 1-й (1), 5-й (2), 7-й (3), 11-й (4), 13-й (5) гармоник тока. ${ }^{4}$

Амплитуда $R_{m k}$ для стального шинопровода, при равномерном изменении расстояния между троллеями, изменяется по нелинейному закону, что обусловлено магнитными свойствами материала.

Максимальное расстояние между стальными троллеями шинопровода, при котором отсутствует влияние внешнего поля от тока соседней троллеи, составляет $l_{\max }=3 l$. Используя полученные зависимости (рис. 4), можно построить поверхностную зависимость амплитуд изменения активного сопротивления от действия внешнего магнитного поля, в зависимости от частоты и расстояния между троллеями шинопровода в функции

\footnotetext{
${ }^{4}$ Appendix 1
}

При увеличении расстояния между соседними троллеями, значение амплитуды изменения активного сопротивления $\left.R_{k}\right|_{A B}$ фазы А от действия стороннего тока в соседнем троллеи условной фазы В шинопровода, для всех $k$ - гармоник тока, будут уменьшаться и стремиться к нулю (рис.3). При этом начальная фаза $\left.R_{k}\right|_{A B}$ для всех $k$-х гармоники тока будет равной $\alpha=0$.

По данным полевого моделирования получены зависимости $R_{m k}$ от расстояния между двумя стальными троллеями (рис. 4) на 1 м их длины для соответствующих значений частоты $k$-ой гармоники тока.

вида $R \mid=f\left(f_{k}, l\right)$ с помощью бикубической интерполяции сплайнами и получить матрицу коэффициентов бикубического полинома. Это позволит определить амплитуду изменения активного сопротивления троллеи условной фазы А от действия внешнего магнитного поля, в зависимости от расстояния между соседними троллеями условных фаз В и С шинопровода для всех спектров частоты, как основной, так и высших гармоник, а также интергармоник тока.

Согласно уравнению (1), с учетом того, что начальная фаза для всех $k$-х гармоник тока активного сопротивления $\alpha=$ const, a активное сопротивление без наличия 
внешнего магнитного поля $\left.R_{k}{ }^{6}\right|_{A A} \quad$ и амплитуда изменения активного сопротивления троллеи $R_{m k}$ от влияния внешнего магнитного поля известны, и определяются с помощью зависимостей (рис. 2) и (рис. 4), можно для каждой фазной троллеи шинопровода, согласно (4)(6) определить значение активного сопротивления.

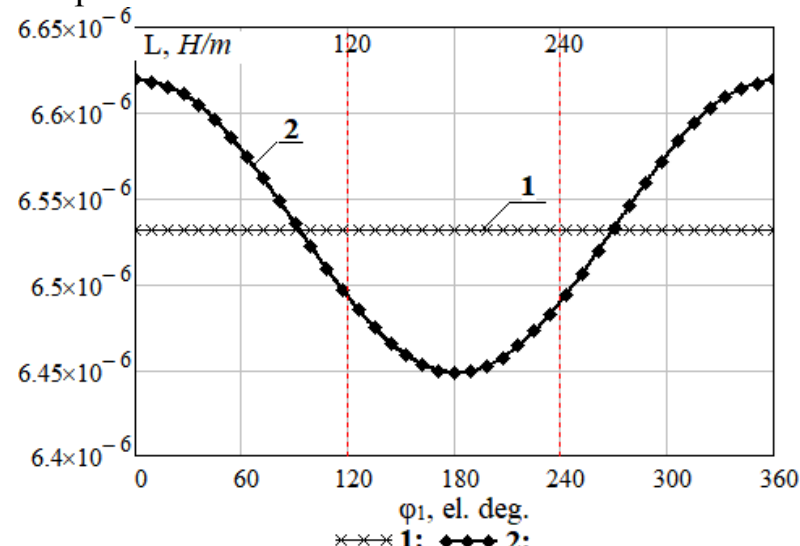

a)

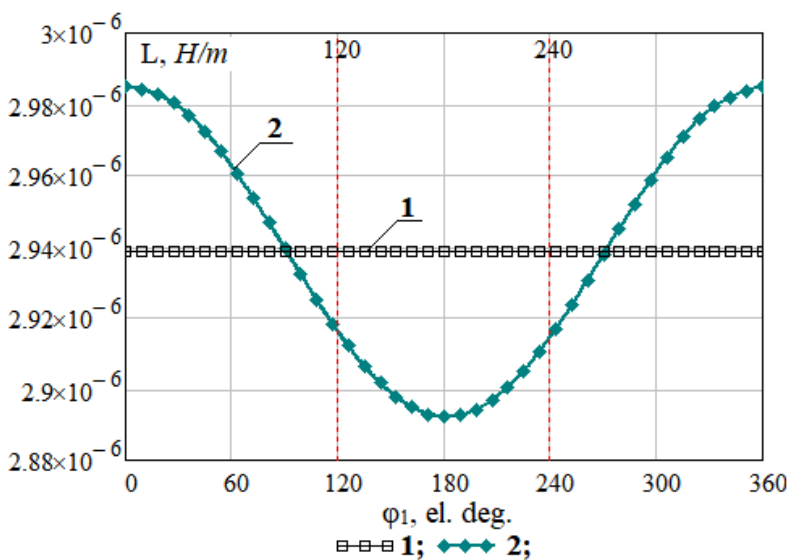

B)

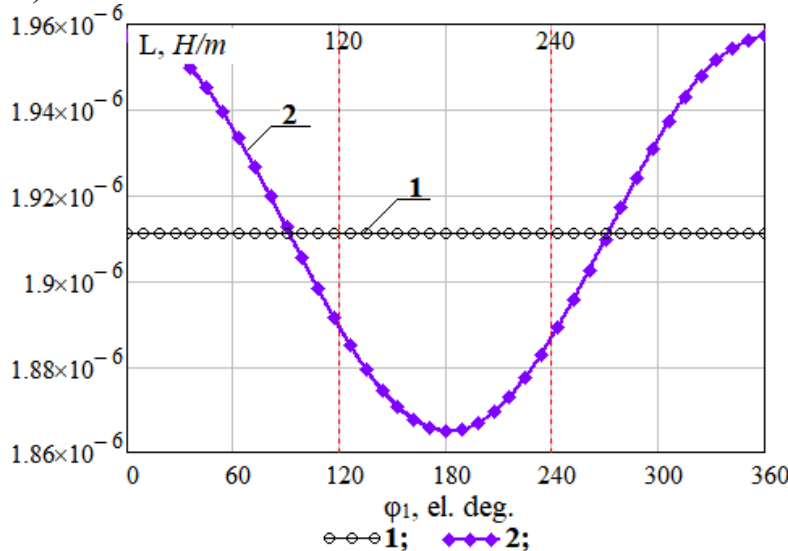

д)
На основе данных полевого моделирования получена зависимость взаимной индуктивности, $\left.M_{k}\right|_{A B}$ стального троллея шинопровода для условной фазы А от угла сдвига $\varphi_{1}$ вектора тока, протекающего в соседней троллее условной фазы В, при соответствующих $k-\mathrm{x}$ гармоник тока в зависимости от расстояния между соседними троллеями (рис. 5).

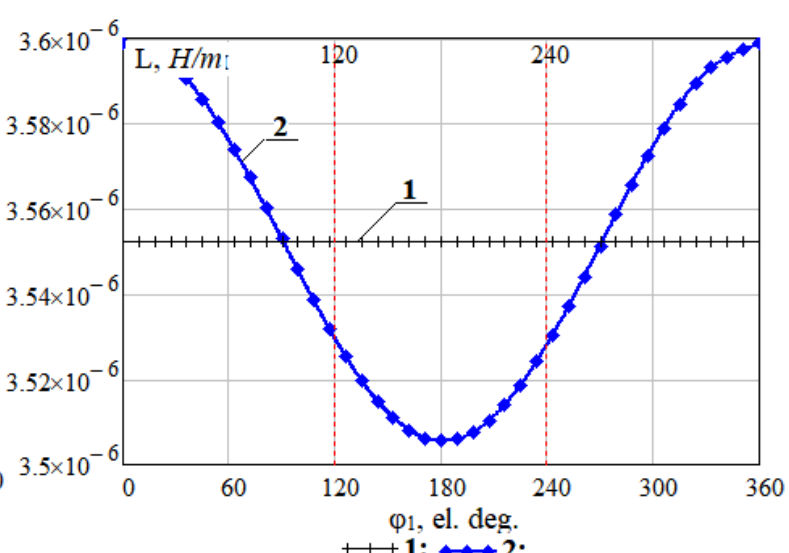

б)

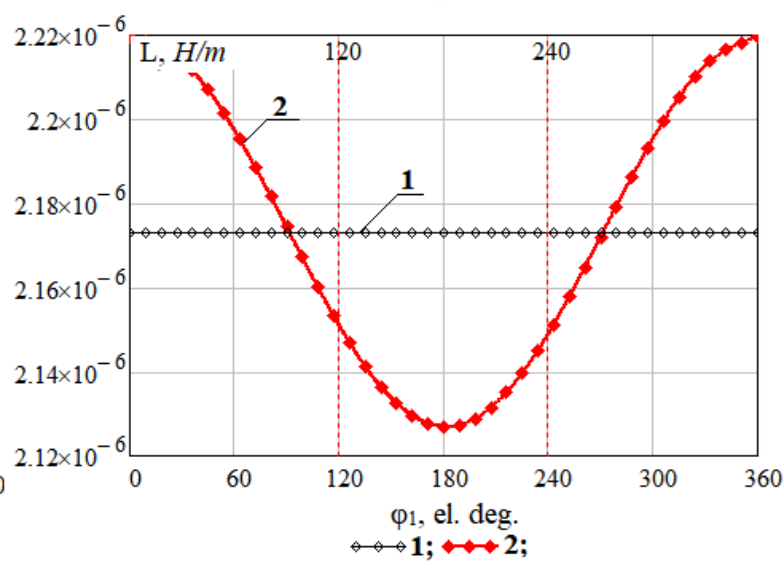

г)

Рис. 5 - Зависимости индуктивности рассеивания (1) и взаимной индуктивности (2) стального троллея шинопровода от угла сдвига вектора тока, протекающего в соседнем троллее для 1-й (а), 5-й (б), 7-й (в), 11-й (г), 13-й (д) гармоник тока.

\section{${ }^{5}$ Appendix 1}


Взаимная индуктивность троллеи условной фазы А будет отсутствовать $\left(\left.M_{k}{ }^{\text {}}\right|_{A B}=0\right)$, при углах сдвига между векторами условной фазы А и В при $\varphi_{1}=90$ эл. град. и $\varphi_{1}=270$ эл. град. При этом взаимная индуктивность фаз $\left.M_{k}\right|_{A B}$ будет опережать активное сопротивление $\left.R_{k}\right|_{A B}$ на угол $\alpha=90$ эл. град. (рис. 3, рис.5).

По данным полевого моделирования получены зависимости амплитуд взаимной

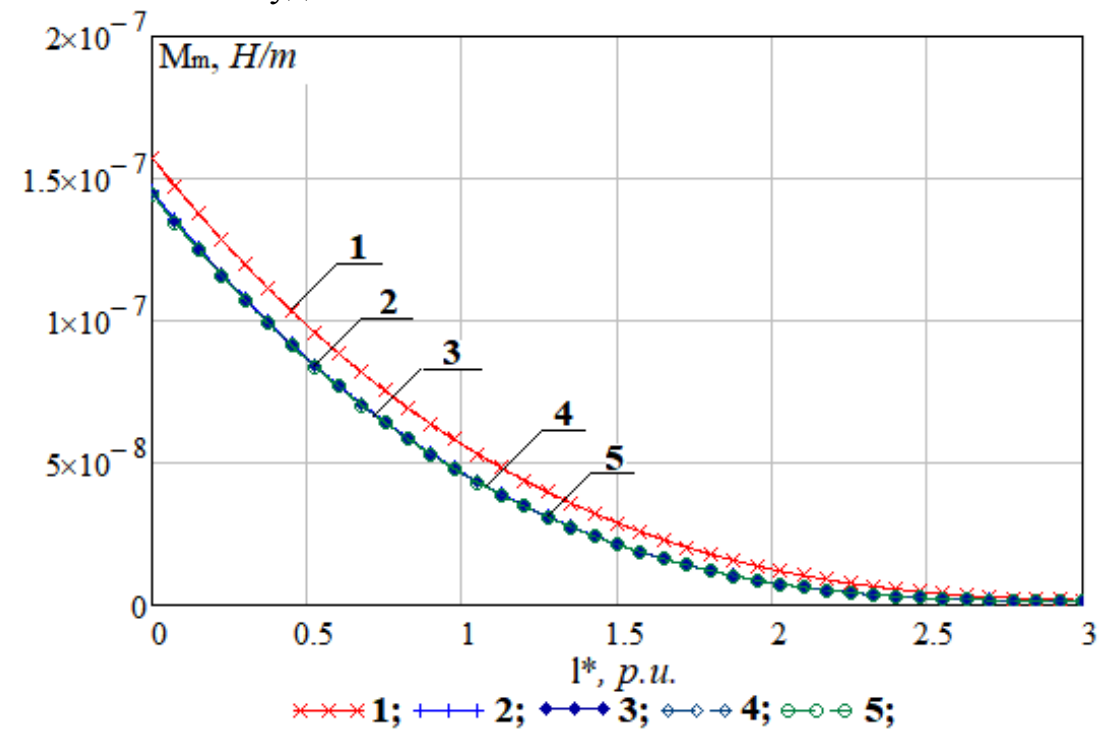

Рис. 6 - Зависимости амплитуд взаимных индуктивностей в зависимости от расстояния между стальными троллеями шинопровода для 1-й (1), 5-й (2), 7-й (3), 11-й (4), 13-й (5) гармоник тока. ${ }^{6}$

Максимальное расстояние между стальными троллеями шинопровода, при котором отсутствует влияние внешнего поля, также составляет $l_{\max }=3 l$. Также, можно построить поверхностную зависимость взаимной индуктивности от частоты и расстояния между троллеями шинопровода в виде функции $M_{m k}=f\left(f_{k}, l^{*}\right)$ c помощью бикубической интерполяции сплайнами и получить матрицу коэффициентов бикубического полинома $M_{m k}$ для соответствующих значений частоты $f_{k} k$-х гармоник тока и расстояния $l$ между соседними троллеями шинопровода.

Согласно уравнению (3) с учетом того, что начальная фаза для всех $k$-х гармоник тока взаимной индуктивности $\alpha$ =const, а индуктивность рассеивания $\left.L_{k}\right|_{\text {AA }}$ и индуктивности $M_{m k}$, в зависимости от расстояния между двумя троллеями (рис. 6) на 1м их длины для соответствующих значений частоты $k$-х гармоник тока. Амплитуда взаимных индуктивностей $M_{m k}$ для стального шинопровода, при равномерном изменении расстояния между троллеями шинопровода, изменяется по нелинейному закону, что также обусловлено магнитными свойствами материала. 
превышает

$\left|\delta \Delta U_{k}\right| \leq 0,02 \%$.

По результирующим потерям напряжения $\left|\delta \Delta U_{k}\right| \leq 0,14 \%$.

По сравнению со значениями потерь напряжения, полученные без учета угла сдвига по фазе $\phi_{T}$, согласно уравнению (16), невязка по значениям потерь напряжения при $f_{1}=50 Г ц\left|\delta \Delta U_{k}\right| \leq 35,97 \%$. и результирующим потерям напряжения при действии высших гармоник тока $\left|\delta \Delta U_{k}\right| \leq 38,61 \%$.
Для подтверждения адекватности предложенного усовершенствованного уравнения (17) при разных значениях коэффициента мощности сети $\cos (\varphi)$ проведена валидация расчетных значений падения напряжения по (17) с результатами испытаний (табл. 1). На рис. 7 показан испытательный стенд троллейного стального шинопровода открытого типа, профиль уголков 20x20x2, длина 1м.

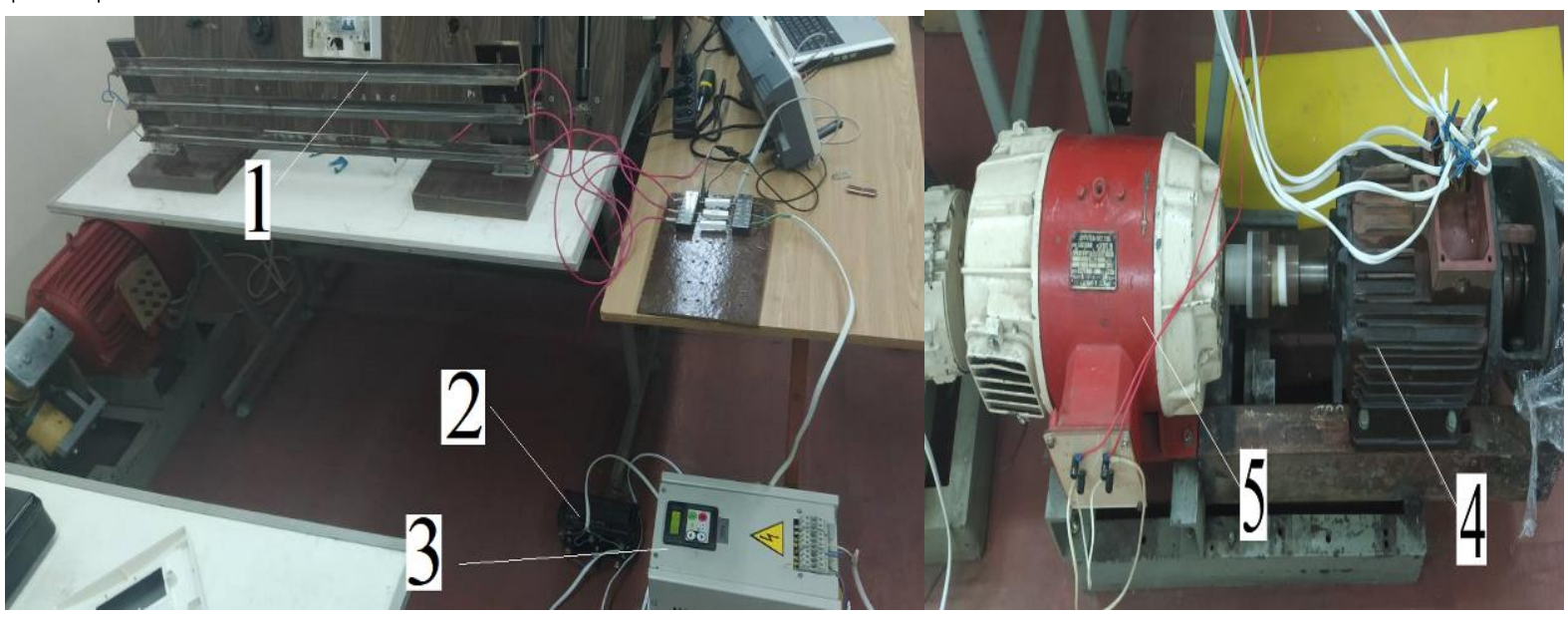

Рис. 7 - Испытательный стенд троллейного стального шинопровода открытого типа.

К началу троллей (1) от трансформатора через кабель подводится напряжение 380B. Концы троллей (1) через входной фильтр (2) и преобразователь частоты (ПЧ) ЭКТ4Д1 25-50УХЛ мощностью 25кВт (3) подключены к асинхронному двигателю, мощностью 2 кВт (4), нагрузкой которому служит двигатель постоянного тока П32 мощностью 3,2кВт (5) (см. рис.7). Коэффициент мощности регулируется изменением нагрузки на двигатель. Пофазное определение коэффициента мощности, а также напряжение на входе и выходе фазных троллеев шинопровода выполнялось с помощью осциллографа OWON XDS3202E. Падение напряжения в фазных троллеях шинопровода проводилось для основной гармоники тока без ПЧ и для результирующего тока с высшими гармониками, генерируемыми в сеть ПЧ. В табл. 1 приведены значения потерь напряжения для фазы А. Для фаз В и С выполнялась симметрия падения напряжения. Отклонение между фазами не
Таблица $1^{8}$

Валидация расчетных и экспериментальных данных потерь напряжения в троллеях шинопровода.

\begin{tabular}{|c|c|c|c|}
\hline$\Delta U$ & Calculation & Testing & $\delta, \%$ \\
\hline \multicolumn{4}{|c|}{$\begin{array}{l}\text { Without frequency converter, } \cos (\varphi)=0,087, \\
\qquad I=1,7 A \text { (idling) }\end{array}$} \\
\hline$\Delta U_{1}, \mathrm{MB}$ & 0,31 & 0,34 & 7,56 \\
\hline \multicolumn{4}{|c|}{$\begin{array}{c}\text { With frequency converter, } K_{m}=0,96 \\
I=1,7 A \text { (idling) }\end{array}$} \\
\hline$\Delta U_{\text {peз }}, \mathrm{MB}$ & 4,34 & 4,7 & 7,62 \\
\hline \multicolumn{4}{|c|}{$\begin{array}{l}\text { Without frequency converter, } \cos (\varphi)=0,378 \\
\qquad I=2,5 \mathrm{~A}\end{array}$} \\
\hline$\Delta U_{1}, \mathrm{MB}$ & 1,84 & 1,95 & 5,75 \\
\hline \multicolumn{4}{|c|}{$\begin{array}{l}\text { With frequency converter, } K_{m}=0,97 \\
I=2,5 \mathrm{~A}\end{array}$} \\
\hline$\Delta U_{p e_{3}}, \mathrm{MB}$ & 26,0 & 27,5 & 5,45 \\
\hline \multicolumn{4}{|c|}{$\begin{array}{l}\text { Without frequency converter, } \cos (\varphi)=0,656 \\
\qquad I=3,6 \mathrm{~A}\end{array}$} \\
\hline$\Delta U_{1}, \mathrm{MB}$ & 5,15 & 5,4 & 4,62 \\
\hline \multicolumn{4}{|c|}{ With frequency converter, $K_{m}=0,98, I=3,6 \mathrm{~A}$} \\
\hline$\Delta U_{p e_{3}}, \mathrm{MB}$ & 73,12 & 76,0 & 3,8 \\
\hline
\end{tabular}

7,8 Appendix 1 
превышало $\quad \delta \Delta U=1,32 \% . \quad$ Погрешность измерительных приборов, согласно паспорту, не превышает $2 \%$.

Таким образом, согласно полученным данным испытаний $\mathrm{c}$ достаточной точностью подтверждается адекватность и эффективность предложенного подхода, который позволяет для каждой соответствующей частоты $k$-х составляющих гармоник тока определить параметры троллеев шинопровода с учетом скин-эффекта, эффекта близости, поверхностных и других краевых эффектов, а также несимметрии по фазе фазных токов, независимо от расположения троллеев, расстояний между ними и количества фаз шинопровода.

\section{ВЫВОДЫ}

Предложен подход, который с высокой точностью и эффективностью, при определенных зависимостях активного и индуктивного сопротивлений, с учетом и без учета действия внешнего магнитного поля, для каждой $k$-й гармоники тока и расстояния между троллеями шинопровода, для симметричных и несимметричных режимов работы токоприемников, а также коэффициента мощности сети, позволяет определить фазные значения активного, реактивного сопротивлений и падение напряжения, независимо от формы и расположения троллеев, расстояния между ними и количества фаз шинопровода. Данный подход без затрат времени на полевое моделирование может быть использован в инженерной практике, что позволит эффективно подобрать конфигурацию сети цехового электроснабжения, определить места установки дополнительных подпиточных секций и шин, точно определить установленную мощность фильтрующих и компенсирующих устройств, что даст возможность повысить качество и надежность работы электроприемников с заявленными паспортными данными, а также повысить коэффициент мощности сети.

Доказано, что активное сопротивление фазного троллея шинопровода, при изменении угла вектора тока на полный его период, протекающего в соседнем троллее, изменяется с одинаковой начальной фазой для всех $k$-х гармоник тока по синусоидальному закону с постоянной составляющей, равной значению активного сопротивления соответствующей частоты $k$-й гармоники тока при отсутствии внешнего магнитного поля. Полная индуктивность фазного троллея шинопровода при изменении угла вектора тока на полный его период, протекающего в соседнем троллее, изменяется с одинаковой начальной фазой для всех $k$-х гармоник тока по косинусоидальному закону с постоянной составляющей, равной значению индуктивности рассеивания троллея соответствующей частоты $k$-й гармоники тока.

Получено аналитическое выражение зависимости между падением напряжения в троллеях шинопровода и коэффициентом мощности сети, которое с высокой точностью и эффективностью позволяет определить потери напряжения в каждой фазе при соответствующих параметрах троллейного шинопровода, в зависимости от коэффициента мощности сети, как для основной, так и с учетом высших гармоник тока, и отличающееся тем, что учитывает угол фазового сдвига падения напряжения, вызванного действием внешнего магнитного поля от токов в соседних троллеях шинопровода, равного $\operatorname{arctg}\left(X_{k} / R_{k}\right)$. Данные экспериментального исследования подтверждают высокую эффективность и точность предложенного подхода.

\section{APPENDIX 1 (ПРИЛОЖЕНИЕ 1)}

Fig. 1. Results of $2 D$ field simulation for steel trolleys of an L-busbar at a frequency of $f_{k}=50 \mathrm{~Hz}$ (a) and $f_{k}=650 \mathrm{~Hz}$ (b) at the corresponding values of the amplitude of current harmonics.

Fig. 2. Dependences of resistance (1) and leakage inductance (2) on frequency of the $k$-th current harmonics in the absence of an external magnetic field.

Fig. 3. Dependences of resistance of phase A steel busbar's trolley on the shift angle of the current vector flowing in the adjacent trolley of phase $B$ for the 1-st (a), 5-th (b), 7-th (c), 11-th (d), 13-th (e) current harmonics.

Fig. 4. Dependences of the amplitudes change in the resistance at the influence of an external magnetic field, depending on the distance between the adjacent trolleys for the 1-st (1), 5-th (2), 7-th (3), 11-th (4), 13-th (5) current harmonics.

Fig. 5. Dependences of the leakage inductance (1) and mutual inductance (2) of the steel busbar's 
trolley at the shift angle of the current vector, flowing in the adjacent trolley for the 1-st (a), 5-th (b), 7-th (c), 11-th (d), 13-th (e) current harmonics.

Fig. 6. Dependences of amplitudes of mutual inductances, with regard to the distance between the steel busbar's trolleys for the 1-st (1), 5-th (2), 7-th (3), 11-th (4), 13-th (5) current harmonics.

Fig. 7. Test stand for open-type busbar's trolleys.

Table 1. Validation of calculated and testing data on voltage drop in busbar's trolleys.

\section{Литература (References)}

[1] Lees, A., et al. Electromechanical Analysis of the Busbar Support System in the LHC Electrical Feed Boxes and their Consolidation with Remotely Installed Clamps. IEEE Transactions on Applied Superconductivity, 201, vol. 26, no. 4, doi:10.1109/TASC.2016.2527746.

[2] Zare, F., Ledwich, G. F. Reduced Layer Planar Busbar for Voltage Source Inverters. IEEE Trans. Power Electron, 2002, Vol. 17, no. 4, pp. 508-516.

[3] Gaoyu, Z., Zhengming, Z., Liqiang, Y. [Study on DC Busbar Structure Considering Stray Inductance for the Back-to-Back IGBT-Based Converter]. "IEEE: Applied Power Electronics Conference and Exposition”. Long Beach, CA, USA, 2013, pp. 1213- 1218. doi: 10.1109/APEC.2013.6520453

[4] Singh, A. K., Patel, S. Mitigation of Inrush Current For Single Phase Transformer by Control Switching Method. International Journal of Electronics, Electrical and Computational System, 2015, Vol. 4, pp. 146150.

[5] Pivnyak, G.G., Zhezhelenko, I.V., Papaika, Yu.A. Estimating Economic Equivalent of Reactive Power in the Systems of Enterprise Electric Power Supply. Naukovyi Visnyk Natsionalnoho Hirnychoho Universytetu, 2016, no.5, pp. 62-66.

[6] Pivnyak, G.G., Zhezhelenko, I.V., Papaika, Yu.A., Lysenko, O.H. Interharmonics in Power Supply Systems. Naukovyi Visnyk Natsionalnoho Hirnychoho Universytetu, 2017, no.6, pp. 109-114.

[7] I. V. Zhezhelenko, Y. L. Sayenko and A. V. Gorpinich, "Electromagnetic Compatibility in the Industrial Electric power Supply Systems," "10th International Conference on Electrical Power Quality and Utilisation”, 2009, pp. 1-6, doi: 10.1109/EPQU.2009.5318838.

[8] Fedorov, A.A. Spravochnik Energetika Promyshlennykh Predpriyatii T. 1. Elektrosnabzheniye [Reference Book Power Engineering of Industrial Enterprises T. 1. Power supply]. Under the general ed. Fedorov
A.A. Moscow, Gosenergoizdat, 1987, p. 840. (In Russian).

[9] Zimin, E.N. Elektrooborudovanie Promyshlennykh Predpriyatii i Ustanovok [Electrical Equipment of Industrial Enterprises and Installations]. Moscow, Energoizdat, 1981, p 552. (In Russian).

[10] Andrienko, P. D., O. V. Nemykina, A. A. Andrienko. High Current Harmonics Influence on the Choice of Conductors of Crane Power Supply Systems. Electrical Engineering \& Electromechanics, 2019 no. 3 pp. 24-29. doi: 10.20998/2074-272X.2019.3.04.

[11] Szczegielniak, T., Piątek, Z., Baron, B., Nski, L., Kusiak, D., Pasierbek, A. A Discrete Numerical Method for Magnetic Field Determination in Three-Phase Busbars of a Rectangular Cross-Section. Turkish Journal of Electrical Engineering and Computer Sciences, 2016, no. 24, pp.1279-1291. DOI: 10.3906/ELK-1310-129

[12] Piątek, Z., Baron, B., Szczegielniak T., Kusiak D., Pasierbek A. Numerical Method of Computing Impedances of a Three-Phase Busbar System of Rectangular Cross Section. Progress In Electromagnetics Research, 2013, Vol. 51, pp. 135-156.

[13] Matsuki, M. Matsushima A. Improved Numerical Method for Computing Internal Impedance of a Rectangular Conductor and Discussions of Its High Frequency Behavior. Progress In Electromagnetics Research, 2012, Vol. 23, pp. 139-152.

[14] Milykh, V.I., Polyakova, N.V. Comparative Analysis of the Variable Magnetic Field on the Surface of the Rotor of Turbogenerators with Different Numbers of Stator Teeth in the Load Condition. Technical Electrodynamics, 2014, no. 2, pp. 29-36.

[15] Bastos, J. P. A, Sadowski, N. Electromagnetic Modeling by Finite Element Methods. Boca Raton: CRC Press, 2003, 510 p. ISBN 9780203911174. doi: $10.1201 / 9780203911174$

[16] Suh, Y., Kim, C. A Study on High-Current Rectifier Systems With Mitigated TimeVarying Magnetic Field Generation at AC Input and DC Output Busbars. "IEEE Transactions on Power Electronics”, 2012, vol. 27, no. 3, pp. 1212-1219. doi: 10.1109/TPEL.2011.2167762.

[17] Bonnet, M., Lefevre, Y., Llibre, J. F., Harribey, D., Defay, F., Sadowski, N. 3D Magnetic Field Model of a Permanent Magnet Ironless Axial Flux Motor with Additively Manufactured Non-Active Parts. "19th International Symposium on Electromagnetic Fields in Mechatronics, Electrical and Electronic Engineering" (ISEF), 2019, pp. 1-2, doi: 10.1109/ISEF45929.2019.9096991.

[18] Zagirnyak, M.V. Determination of the Pon- 
deromotive Magnetic Force When Calculating the Field by the Conformal Transformation Method, Technical Electrodynamics, 2020, no.3, pp. 9-14.

[19] Yamazaki K., Kakoo S., Utsunomiya R., Morishita D., Ohto, M. Characteristics Analysis of Large Induction Motors Considering Core Ends and Duct Spaces by Using 3D Finite Element Method,"23rd International Conference on Electrical Machines and Systems (ICEMS), 2020, pp. 1199-1203, doi: 10.23919/ICEMS50442.2020.9291111.

[20] Pekel, U., Mittra, R. A Finite Element Method Frequency Domain Application of the PML Concept. Microwave and Optical Tech. Let,

\section{Сведения об авторах.}

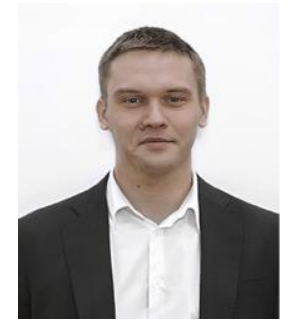

Коцур Михаил Игоревич, доцент, кандидат технических наук, доцент кафедры электрических и электронных аппаратов НУ «Запорожская политехника», Украина. E-mail: kotsur8@gmail.com Область научных интересов: исследования электромагнитных, энергетических и тепловых процессов электромеханических комплексах и системах.

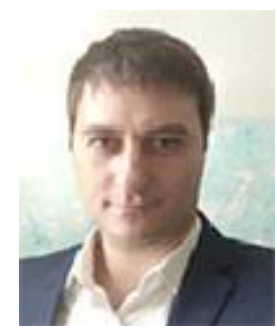

Ярымбаш Дмитрий Сергеевич, профессор, доктор технических наук, заведующий кафедрой электрических машин НУ «Запорожская политехника», Украина.

E-mail: yarymbash@gmail.com Область научных интересов: совершенствование электротехнологических процессов и режимов работы электротехнических комплексов промышленных предприятий.
1995, Vol. 9 (no. 3), pp. 117-122.

[21] Paoli, G., Biro, O. Time Harmonic Eddy Currents in Non-Linear Media. "COMPEL» - The International Journal for Computation and Mathematics in Electrical and Electronic Engineering., 1997, vol. 17, no. 5. - pp. 567-575.

[22] IEC (61000-3-12: 2004) Electromagnetic compatibility of technical means. Limit of harmonic current components created by technical means with a current consumption of more than $16 \mathrm{~A}$, but not more than $75 \mathrm{~A}$ (in one phase), connected to low-voltage generalpurpose power systems. Norms and methods of testing.

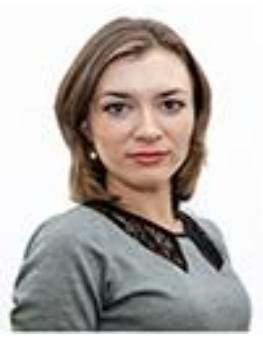

Безверхняя

Юлия Сергеевна, аспирант кафедры электрических и электронных аппаратов НУ «Запорожская политехника», Украина. Еmail: juliaeea@urk.net Область научных интересов: оптимизация структуры и параметров электрических сетей по критерию энергоэффективности.

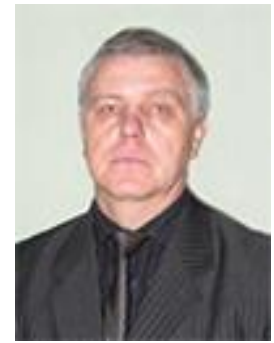

Коцур Игорь Михайлович, доцент, кандидат технических наук, доцент кафедры электрических машин НУ «Запорожская политехника», Украина. Еmail: $\quad$ kotsur@zp.edu.ua Область научных интересов: электромагнитные, электромеханические и энергетические процессы в электрических машинах. 